Revista Científica de FAREM-Estelí

Medio ambiente, tecnologia y desarrollo humano

Año 10 | Edición especial: artículos de revisión documental

ISSN: 2305-5790

https://rcientificaesteli.unan.edu.ni

DOI: https://doi.org/10.5377/farem.v0i0.11615

\section{Disponibilidad y adaptabilidad de los recursos hídricos en la Subcuenca Aguas Calientes (Somoto-San Lucas), Madriz}

\section{Availability and adaptability of water resources in the Aguas Calientes sub-watershed (Somoto - San Lucas), Madriz}

\section{Rolando José Gutiérrez Corea}

Estudiante de doctorado en Gestión y Calidad de la Investigación Científica Il Cohorte 2020-2023 (UNAN-Managua). Docente de Biología y Oficial de Investigación, UNAN León, CUR Somoto. https://orcid.org/0000-0001-9087-6118

rolando.gutierrez@curs.unanleon.edu.ni
RECIBIDO

$26 / 03 / 2021$

ACEPTADO

19/052021

\section{RESUMEN}

Este artículo tiene como objetivo el estudio sobre la disponibilidad y adaptabilidad de los recursos hídricos en la Subcuenca Aguas Calientes (Somoto - San Lucas), departamento de Madriz. La metodología utilizada fue una revisión de literatura documental, con recogida de datos en bases electrónicas como: E-libro, Proquest y repositorio del $\mathrm{CNU}$, basado en el modelo de cogestión adaptativa de cuencas hidrográficas. Entre las estrategias de adaptación se encontraron la recuperación del bosque, aplicación de tecnologías para captación y almacenamiento de agua de lluvia, protección de las fuentes hídricas y asociaciones de productores como microempresas. En los resultados de los estudios se indican estrategias y tecnologías que aplican los productores agropecuarios para adaptarse a la variabilidad climática y a la sequía. Por otro lado, aborda la adopción del enfoque de cuencas como herramienta de planificación de los gobiernos municipales que tiene una importancia estratégica debido a la integración de las variables medio ambientales, principalmente en cuanto a las técnicas para favorecer la conservación de suelos y aguas. Se plantea un cambio en el manejo de la Sub cuenca como: la diversificación, la factibilidad financiera de dos cultivos adaptados a la sequía y producidos en la subcuenca como el henequén y la pitahaya.
PALABRAS CLAVE

Disponibilidad; adaptación; recursos hídricos; subcuenca; modelo de cogestión. 


\section{ABSTRACT}

The objective of this paper is to study the availability and adaptability of water resources in the Aguas Calientes sub- watershed (Somoto - San Lucas) department of Madriz. The methodology used was a documentary literature review, with data collection in electronic databases such as: E-libro, proquest and CNU repository, based on the model of adaptive co-management of watershed. Adaptation strategies included forest recovery, application of technology for rainwater harvesting and storage, protection of water source and producer associations as a micro-enterprise. The results of the studies indicate strategies and technologies applied by agricultural producer to adapt to climate variability and drought. On the other hand, it considers the adoption of the watershed approach as a planning tool for municipal government, which is of strategy importance due to the integration of the environment variables, mainly in terms of techniques to favor soils and water conservation. A change in the management of the sub-watershed is proposed, such as: diversification, financial feasibility of two crops adapted to drought and produced in the sub- watershed as henequen and pitahaya.

\section{KEYWORDS}

Availability; adaptation; water resources; sub-watershed; comanagement model. 


\section{INTRODUCCIÓN}

Para Solomon (2013) define que las "adaptaciones son características hereditarias que potencian la capacidad de un organismo para sobrevivir en un ambiente en particular" (p. 5). Tradicionalmente disponibilidad y calidad de los recursos hídricos en la Subcuenca Aguas Calientes (Somoto - San Lucas), departamento de Madriz, requiere de adaptabilidad lo que trae consigo cambios en la especie más que en los organismos individuales, muchas adaptaciones se adquieren a través de grandes períodos evolutivos.

Para Parra (2009) el agua es patrimonio de la humanidad, como elemento de la naturaleza y como recurso indispensable para las actividades económicoproductivas. El uso y manejo de las fuentes hídricas es un reto para las instituciones, las autoridades, los grupos sociales y demás entes responsables de la conservación de la calidad del agua en diversos sistemas naturales por lo que se pretende realizar un estudio de carácter documental sobre tecnologías y estrategias que garanticen la permanencia y optimización del agua.

Toledo, Barragán y Ortiz (2005) consideran a una cuenca hidrológica, como un sistema, debido a que es unidad espacial ecogeográfica, la cual está integrada por una alta diversidad de patrimonios naturales y culturales, que reúnen condiciones especiales y que permiten analizar procesos ecológicos y humanos de larga duración. La cuenca es un sistema complejo, dinámico y abierto, cuyos componentes naturales y culturales se encuentran estrechamente interconectados.

La subcuenca del río Aguas Calientes se ubica en la región de Las Segovias, departamento de Madriz, presenta gran variabilidad de precipitaciones, un periodo anual de sequía generalmente mayor de seis meses y ciclos caniculares irregulares; la principal actividad agrícola es la producción de granos básicos (MARENA, 2003). Por tal razón se debe de recurrir a estrategias de autogestión de los recursos hídricos, mediante el control de actividades como la extracción de maderera, el avance de la frontera agrícola y ganadera.

Hace algunos años este recurso cubría las necesidades de la población durante la época seca, pero debido a los efectos del cambio climático las sequías se han prolongado, ocasionando pérdidas de los ciclos agrícolas, aparición de enfermedades, pobreza y explosión social en demanda del vital líquido. En vista de esta situación se pretende desarrollar un estudio documental sobre el análisis de tecnologías y estrategias que garanticen la disponibilidad y adaptabilidad del recurso en 10 comunidades de la subcuenca para mejorar las condiciones de 10 comunidades de la subcuenca Aguas Calientes. 
Investigaciones recientes, sugieren complementar la perspectiva tradicional, situando el tema del agua en una visión global, pues la mayoría de las economías están involucradas en el intercambio internacional de bienes y servicios, influyendo sobre los patrones de uso de agua doméstica.

Padrana (2018) considera que es de vital importancia realizar un estudio sobre la gestión del agua, recurso indispensable para los seres vivos de nuestro planeta presente en todas las formas de vida y en mucha de las actividades que el hombre desarrolla para su subsistencia como la agricultura, ganadería y procesos de obtención de energía según los estudios realizados.

Padrana (2018) afirmó que un sistema de gestión de agua tiene que dar respuestas a varios problemas que enfrentan los pobladores de la subcuenca Aguas Caliente como: Garantizar uso sostenible, proteger y conservar su calidad, tanto para humano como ecológica, satisfacer las demandas para un desarrollo social aceptable (p. 23).

Es importante señalar que este estudio tiene la finalidad de documentar a través de una revisión bibliográfica la gestión y disponibilidad de los recursos hídricos en la Subcuenca Aguas Calientes bajo el modelo de cogestión adaptativa de cuencas hidrográficas.

A continuación, se hará reflexión sobre el modelo de cogestión adaptativa que visualiza las cuencas hidrográficas como sistemas integrales de flujos hídricos de interés público y colectivo. Dicho modelo promueve el ordenamiento institucional a través de la gobernanza local y de los mecanismos de organización y participación de las organizaciones locales en la toma de decisiones.

Esta investigación documental reconoce la importancia del modelo de cogestión en la Subcuenca Aguas Calientes, es vital para la disponibilidad y gestión de los recursos hídricos, parte de la necesidad de asegurar la cantidad y calidad del agua por medio de plataformas de concertación que impulsen el encuentro y diálogo entre los actores locales para desarrollar una agenda territorial común en la se complementen esfuerzos y recursos.

Marin, Fernandez, Martínez y Suárez (2006) plantean que: La gestión y administración adecuada de los recursos hídricos, que garantizan su uso sostenible, obliga a conocer su comportamiento y su respuesta ante las diferentes intervenciones antrópicas; por lo cual, se hace importante la implementación de metodologías rápidas y económicas para la evaluación de las características de las fuentes de agua que permitan determinar, de una manera aproximada, su calidad y sus posibles usos.

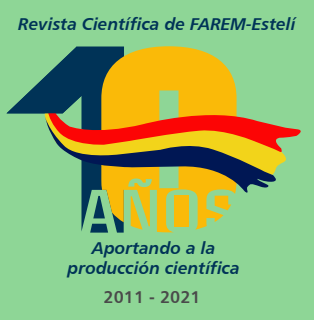




\section{MATERIALES Y MÉTODOS}

Este estudio es resultado de una exhaustiva revisión documental de diferentes recursos bibliográficos sobre el tema disponibilidad y gestión de los recursos hídricos en la subcuenca Aguas Calientes del departamento de Madriz. Se utilizó variedad de estrategias para acceder a los datos y se contó con los criterios de selección de la información como literatura clásica de libros, artículos científicos y tesis publicados en los últimos cinco años, con acceso a los textos completos en digital.

Las principales fuentes consultadas fueron libros, artículos científicos y tesis localizados a través de las bases de datos del repositorio del CNU y de la UNAN - Managua como: E-Libro y Proquest. La estrategia de búsqueda de información consistió en la revisión de estudios de alto nivel científico que proporcionaron datos relevantes sobre el tema. Para facilitar la selección e indagación se creó una base de datos, con la finalidad de resumir información de los artículos, libros o tesis que atendieron a los criterios de inclusión.

Los criterios de selección de la información científica se basaron en las características de los estudios, los cuales responden a la problemática, desde diferentes ámbitos y que tienen una estrecha relación con el problema actual. Estos criterios permitieron ordenar el cuerpo teórico, teniendo en cuenta diferentes aspectos: Relevancia, naturaleza de sus contenidos (datos estadísticos, biográficos, bibliográficos, legislativos, otros), la experiencia en investigación de los autores, la actualización de los estudios, la autenticidad, formato, origen, idioma y accesibilidad.

Los criterios para la calidad de una investigación dependen de las necesidades reales de las personas; sin esa suposición, el isomorfismo de los hallazgos con la realidad no puede tener ningún sentido, es imposible plantear una generalización estricta dentro de una población madre, no puede evaluarse la estabilidad para la investigación de un fenómeno si el fenómeno mismo está sujeto a cambios, y no puede lograr una objetividad, ya que no hay nada de lo cual "distanciarse". 


\section{RESULTADOS}

Los principales estudios para sustentar este artículo documental fueron:

1. Kammerbauer, León, Castellón, Gómez y Prins (2011) realizaron estudio sobre: Evaluación de la adaptación de los productores a la variabilidad climática, principalmente a la sequía, en cuencas hidrográficas de América Central. Parte 2. Estudio de caso en la subcuenca del río Aguas Calientes, Nicaragua.

Esta propuesta permitió identificar y caracterizar las estrategias y tecnologías que aplican los productores agropecuarios para adaptarse a la variabilidad climática y a la sequía. En general, las estrategias y tecnologías empleadas para adaptarse a la sequía no se relacionan con el estrato de la cuenca; algunas sí dependen de la zona de la cuenca, como el empleo de sistemas de riego, captación y almacenamiento de agua, uso de barreras vivas o muertas, abonos verdes y plantación de frutales. Como alternativa de diversificación, se determinó la factibilidad financiera de dos cultivos adaptados a la sequía y producidos en la subcuenca el henequén y la pitahaya.

2. Banegas, Jiménez, Locatelli, Faustino y Campos (2007) en su estudio plantean un modelo de cogestión adaptativa de cuencas hidrográficas. Propuesta conceptual basada en la revisión crítica de las experiencias en Honduras y Nicaragua.

Esta experiencia plantea la hipótesis global del modelo de cogestión que parte de la necesidad de asegurar la cantidad y calidad del agua por medio de plataformas de concertación que impulsen el encuentro y diálogo entre los actores locales para desarrollar una agenda territorial común en la se complementen esfuerzos y recursos. Una de las finalidades es la construcción de acuerdos y arreglos institucionales con una visión territorial, para el desarrollo de capacidades locales que favorezcan la implementación de prácticas y tecnologías amigables con el ambiente.

\section{Localización y características de la subcuenca}

La subcuenca del río Aguas Calientes se ubica en la región de Las Segovias, departamento de Madriz, entre las coordenadas $13^{\circ} 24^{\prime} 10^{\prime \prime}$ y $13^{\circ} 29^{\prime} 28^{\prime \prime}$ latitud norte y $86^{\circ} 34^{\prime} 12^{\prime \prime}$ y $86^{\circ} 39^{\prime} 39^{\prime \prime}$ longitud oeste (Alcaldía Municipal de Somoto 2001). La subcuenca forma parte de la red del río Coco y posee numerosos tributarios. En el área de estudio hay diez comunidades, ocho del municipio de Somoto (Aguas Calientes, Quebrada de Agua, Mansico, Los Copales, Santa Rosa, Rodeo No. 2, Santa Isabel, Uniles) y dos del municipio de San Lucas (El Volcán y El Porcal). La subcuenca abarca una superficie de 47,4 km2 (4737 ha) en los municipios de Somoto (84,5\%) y San Lucas 
(15,5\%). La altitud varía de 620 a 1730 msnm, aunque el $70 \%$ del área está entre 620 y 800 msnm (Cajina, 2006).

Fig. 1. Mapa de localización de las zonas de recarga hídrica de las fuentes de agua.
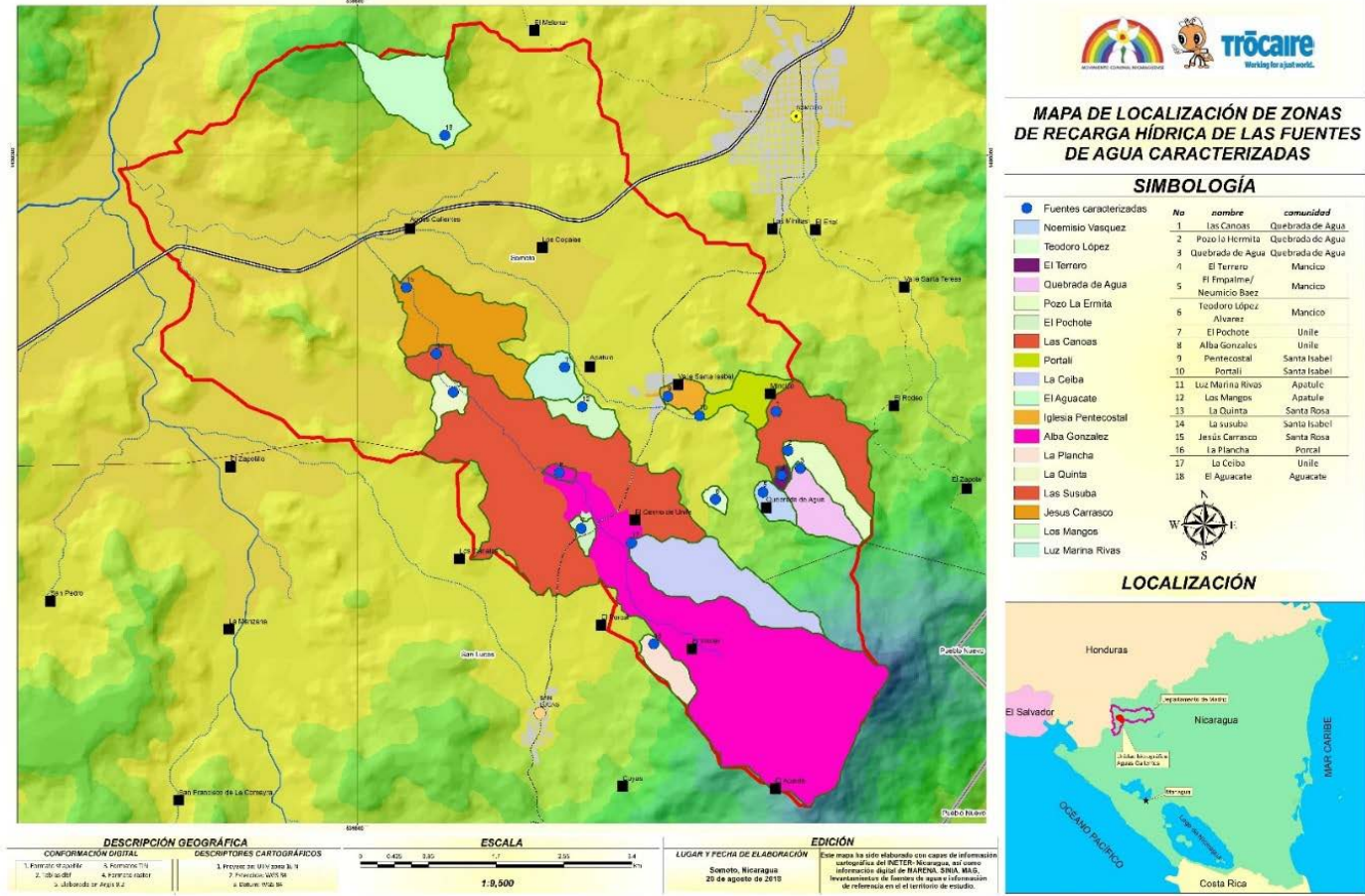

Fuente: Instituto nicaragüense de estudios territoriales, INETER, 2018.

Según INETER (2012) El clima de la subcuenca es tropical seco. La precipitación media anual de los últimos 43 años es de $823 \mathrm{~mm}$ con variaciones entre $448 \mathrm{~mm}$ y $1449 \mathrm{~mm}$. A través del año, las lluvias son muy irregulares en cantidad e intensidad. En general se dan dos periodos estacionales: un periodo seco desde diciembre hasta abril y un periodo lluvioso de mayo a noviembre; en este último entre julio y agosto se presenta un subperiodo de bajas precipitaciones (y a veces totalmente seco) llamado canícula, con duración variable de 15 a 40 días.

\section{Situación ambiental de las cuencas hidrográficas de Nicaragua}

En Nicaragua, la deforestación ha jugado un papel importante en la degradación de las cuencas hidrográficas, lo que a su vez ha sido la causa principal de la erosión hídrica. Este proceso de deterioro ocasiona alteraciones al régimen hidrológico de las cuencas y en sus patrones de drenaje. Se estima que en Nicaragua han sido afectadas por una erosión de fuerte a severa, aproximadamente unos 1,12 millones de hectáreas (MARENA, 2003).

La subcuenca Aguas Calientes (Somoto - San Lucas), es la principal fuente de producción de agua para consumo humano, irrigación y uso animal. La disminución de la calidad y cantidad de las fuentes de agua es una 
preocupación de los gobiernos municipales antes mencionados y de las entidades involucradas en el manejo del recurso agua.

La disminución de las amenazas de contaminación de las fuentes, se convierten en ejes centrales para impulsar programas de comanejo del área hidrológica, con la participación activa y consciente de la población, siendo la finalidad contribuir a formular lineamientos para ejecutar acciones que permitan un mejor uso de los recursos existentes en la subcuenca.

\section{Manejo integrado de cuencas}

El manejo integrado de cuencas es un tema relativamente nuevo y hoy en día la mayoría de los profesionales de diferentes campos están reconsiderando ya este tema como un componente enriquecedor y facilitador de la visión de trabajo. El manejo de cuencas se considera que es un proceso iterativo de decisiones sobre los usos y las modificaciones a los recursos naturales dentro de una cuenca. Así mismo, implica la formulación y desarrollo de actividades que involucran a los recursos naturales y humanos de la cuenca. Por otra parte, el manejo de cuencas conlleva a la participación de la población en los procesos de planificación, concertación y toma de decisiones. Por lo tanto, implica el desarrollo de capacidades locales que faciliten la participación (Jiménez, 2005).

\section{Uso actual de la subcuenca}

Las condiciones de abastecimiento de agua a la población se podrían mejorar formando los comités de agua y saneamiento en las comunidades, involucrando a la población para implementar planes de reforestación en las zonas de recarga y medidas de protección en las fuentes de agua para promover la Gestión Integrada de los Recursos Hídricos. Las principales actividades productivas son la producción de granos básicos para auto consumo, henequén y su transformación, hortalizas, café, fabricación de adobes, tejas y artesanía de barro. La elevación media de la subcuenca es de aproximadamente 730 msmn (Castellón, 2005).

El 70\% del área se ubica en un rango de elevación comprendido entre los 620 y 800 msnm, y el restante $30 \%$ en áreas comprendidas entre 800 a $1730 \mathrm{msnm}$. En la subcuenca se localiza la montaña TEPEZOMOTH la cual es una de los 19 neblíes selvas de altura de hoja ancha de Nicaragua. En esta subcuenca se encuentra un cuarto de la población total de Somoto y es la mayor concentración demográfica rural del municipio: diez comunidades con 7.000 habitantes, aproximadamente.

La adopción del enfoque de cuencas como herramienta de planificación de los gobiernos municipales tiene una importancia estratégica debido a la integración de las variables medio ambientales en el corazón de la metodología, además de inducir a la gestión del consenso y la construcción de alianzas

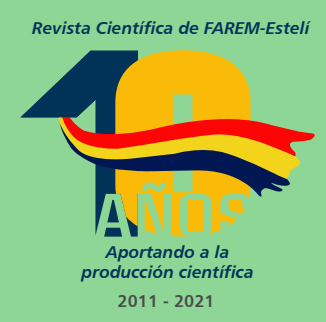


locales. La aplicación de herramientas diversas a la gestión de microcuencas, ofrece la posibilidad de identificar actividades y recomendaciones para su aplicación, incluyendo aquellas utilizadas para la divulgación y actividades didácticas. (Castellón, 2005).

\section{El proceso de Intervención}

Castellón (2005) contempla el diseño del proceso metodológico a seguir con los pobladores y entidades locales para su involucramiento en el proceso. Además del rubro que contempla el Plan Rector es necesario contemplar actividades que se puedan desarrollar en el proyecto a fin de que las comunidades comiencen a emprender acciones.

\section{Agenda territorial común: una guía de acción en la plataforma}

Kammerbaver et al. (2008) propone una agenda común o plan de cogestión como la base para la concertación de una visión territorial colectiva. "Esta agenda no es un instrumento de planificación oficial, sino una herramienta flexible que refleja los acuerdos generados entre las organizaciones participantes en un arreglo de cogestión".

Banegas et al. (2007) plantea que debe de existir una plataforma de concertación logre implementar la agenda territorial común, se debe contar con condiciones mínimas de financiamiento. Puede ser un financiamiento descentralizado y autónomo, o aportes de cada actor institucional.

\section{Aprendizajes conjuntos: acción-investigación}

El contexto de los sistemas ecológicos y sociales se caracteriza por un alto grado de incertidumbre. Por ello, el atributo adaptativo del modelo de cogestión requiere de mecanismos de retroalimentación a través de procesos colectivos de aprendizajes que integren el conocimiento técnico y científico, popular y ancestral de todos los actores involucrados (Banegas et al; 2007).

Las decisiones se toman a partir de consensos sociales, en los que el equipo de facilitación y los participantes tienen poder de decisión. A pesar de ser procesos bastantes imperfectos, son mejores que otros procesos de toma de decisión en los que actores específicos toman decisiones de manera aislada. Los conocimientos se generan en forma iterativa e incremental en una espiral de acción-reflexión y de responsabilidad compartida. Las múltiples perspectivas de los actores en cogestión permiten desarrollar aprendizajes y propuestas innovadoras (Banegas et al; 2007).

El proceso de intervención contempla el diseño del proceso metodológico a seguir con los pobladores y entidades locales para su involucramiento en el proceso. Además del rubro que contempla el Plan Rector es necesario 
contemplar actividades que se puedan desarrollar en el proyecto a fin de que las comunidades comiencen a emprender acciones.

\section{El modelo de cogestión adaptativa de cuencas hidrográficas}

Faustino y Jiménez, (2005) plantean "la cogestión adaptativa de cuencas como un estilo de gestión compartida basada en la intervención experimental, la observación y reflexión de los resultados de las acciones, el aprendizaje continuo, la retroalimentación y reajuste de acciones y métodos, a la luz del conocimiento adquirido con la acción reflexionada". Según los mismos autores, se actúa en forma experimental para generar una mayor claridad sobre cómo realizar los cambios deseados.

La acción-investigación y las alianzas de aprendizaje son un apoyo fundamental para la implementación de la cogestión adaptativa en las cuencas hidrográficas, y están estrechamente relacionadas con la sistematización y el aprovechamiento de las experiencias. El manejo adaptativo de cuencas busca cimentar la gobernabilidad a partir del gobierno local en sus funciones de autoridad y articulador de los actores y grupos de interés (Kammerbauer et al; 2011 ).

El modelo parte del impacto en la calidad y cantidad de agua como finalidad última del manejo de la cuenca, como indicador de la eficacia de las acciones colectivas y como constructor de legitimidad a partir de la convergencia de intereses y la concertación en situaciones de conflictos por el agua (Kammerbauer et al; 2011).

Kammerbauer et al. (2011) se refiere a la propuesta del modelo que visualiza un punto de partida central. La finalidad de todo actuar en el manejo de la cuenca es incidir en el flujo del agua, con el fin de garantizar el abastecimiento continuo y de calidad. Las acciones que se implementen buscan propiciar cambios positivos y medibles que mejoren la calidad de vida de la población.

La dificultad consiste en determinar las tendencias de cambio, ya que el ciclo hidrológico es influido por una serie de factores externos que requieren de mediciones y análisis de series en el tiempo para establecer correlaciones directas entre causa y efecto. En general, en la determinación de flujos se usan apreciaciones rápidas de campo y se correlacionan con la precipitación y el manejo de las zonas de recarga hídrica.

\section{Mecanismos de concertación: plataformas para buscar convergencia y complementariedad}

Kammerbaver et al. (2011) fundamenta que la cogestión adaptativa se relaciona directamente con la gobernabilidad. Los arreglos de cogestión son flexibles y se adaptan a las condiciones cambiantes y específicas del lugar, desde la base local hasta escalas mayores (municipio, subcuenca, etc.). Una

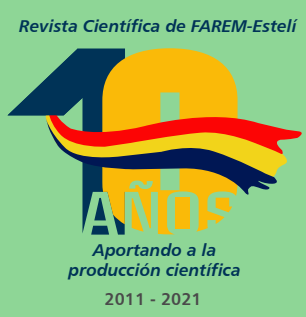


de las condiciones necesarias son los mecanismos de convergencia entre los principales actores y grupo de interés en espacios de concertación. En las plataformas formales e informales, los grupos de interés y los actores locales se vinculan para construir una visión territorial y una agenda común.

A nivel local, diferentes estructuras locales pueden asumir estas funciones, mientras que a nivel de un municipio, subcuenca $u$ otra unidad territorial mayor, la instalación de estos mecanismos requiere de algún grado de formalización (ordenanza municipal o personería jurídica). La participación equitativa y representativa de todos los actores: hombres, mujeres y jóvenes; etnias; pobres, ricos y grupos marginales es clave para la credibilidad y eficiencia de estos espacios. Detalles de las experiencias vividas por los comités de cuenca o sus equivalentes se pueden consultar en (Castellón y Prins, 2009).

\section{Estrategias y tecnologías de adaptación a la sequía}

Se encontraron algunas estrategias y tecnologías que mostraron dependencia de la zona de la cuenca; entre ellas: empleo de sistemas de riego (parte baja), sistemas de captación y almacenamiento de agua, uso de barreras vivas o muertas, empleo de abonos verdes y plantación de frutales (parte alta). Estas estrategias de adaptación corresponden, en el caso del riego, a la principal actividad agrícola producción de hortalizas con microrriego (Cajina, 2006).

Para Cajina (2006) las tecnologías que dependen de la zona indican que para la difusión y utilización de las mismas no se está siguiendo un enfoque integral de cuencas en todos los estratos, principalmente en cuanto a las técnicas para favorecer la conservación de suelos y aguas (barreras vivas o muertas, abonos verdes y sistemas de almacenamiento y captación de aguas). Sin embargo, el hecho de que los sistemas de riego y plantación de frutales sean diferenciados por estratos no indica necesariamente que el enfoque de cuencas esté aislado, sino que las técnicas se adoptan en función de la capacidad de uso del suelo.

Estos resultados coinciden en parte con los obtenidos por (Gómez, 2003) quien determinó un bajo porcentaje de familias que poseen estructuras para almacenar agua, pozos y riego en sus fincas, lo que hace que el grado de vulnerabilidad a la sequía sea muy alto en varias zonas de la subcuenca.

Los cultivos para adaptarse a la sequía no se relacionan con el estrato de la cuenca; algunas sí dependen de la zona de la cuenca, como el empleo de sistemas de riego, captación y almacenamiento de agua, uso de barreras vivas o muertas, abonos verdes y plantación de frutales.

Como alternativa de diversificación, se determinó la factibilidad financiera de dos cultivos adaptados a la sequía y producidos en la subcuenca el henequén 
y la pitahaya. La producción de henequén bajo el sistema tradicional de la subcuenca es una actividad económicamente poco sostenible. El cultivo de pitahaya, en todos los escenarios estudiados, arrojó indicadores financieros que harían atractiva la inversión.

\section{CONCLUSIONES}

En conclusión, este artículo abordó el tema sobre la disponibilidad y adaptabilidad de los recursos hídricos en la subcuenca Aguas Calientes, por lo que permitió documentar las tecnologías y estrategias del modelo de cogestión destacando que la zona posee una baja adaptación a la sequía.

Entre las estrategias de adaptación a la sequía en la subcuenca se encontraron la recuperación del bosque, la aplicación de tecnologías de captación y almacenamiento de agua de lluvia, la protección de fuentes de agua y las asociaciones de productores como microempresas.

Las experiencias descritas evidencian que las actividades productivas como la agricultura y ganadería han contribuido a agudizar el problema de la escasez del agua, debido a que las áreas de bosques están siendo afectadas.

En la subcuenca del río Aguas Calientes se documentó que se aplica un enfoque sistémico de cuencas hidrográficas de manera parcial y con muchas debilidades. Por lo que se necesita realizar coordinaciones para fortalecer las acciones en la búsqueda de la solución a los problemas que presentan los recursos hídricos.

Se plantea en los estudios la adopción de un enfoque de cuencas, como herramienta de planificación de los gobiernos municipales que tiene una importancia estratégica debido a la integración de las variables medio ambientales, principalmente en cuanto a las técnicas para favorecer la conservación de suelos y aguas.

Se propone la implementación del cultivo de pitahaya como una alternativa de adaptación en la subcuenca si se emplea el sistema (mayor número de plantas por superficie), este sistema garantizaría mayor optimización del agua ya que la siembra de este cultivo es resistente a la sequía garantizando la producción y el comercio local.

Es necesario una creciente articulación entre niveles de intervención para la toma de decisiones. La gestión territorial ha permitido que se integren los decisores en varios niveles de decisión: el nivel comunitario, municipal, intermunicipal y nacional. 


\section{REFERENCIAS BIBLIOGRÁFICAS}

Banegas, L., Jiménez, F., Locatelli, B., Faustino, J., \& Campos, M. (2007). Evaluación de la adaptación de los productores. Recursos Naturales y Ambiente, 7.

Castellón, N. (2005). Gestión de Microcuencas como Estrategia de Planificación del Desarrollo Rural. Somoto, Nicaragua.

Cajina, M. (2006).Alternativas de captación de agua en la subcuenca del río Aguas Calientes para mejorar los beneficios socioeconómicos y ambientales de las comunidades de los municipios de Somoto y San Lucas, Nicaragua. Tesis Mag. Sc. Turrialba, CR, CATIE. 228 p.

Endeman C, y. J. (2002). Paradigmas en competencias en la investigación cualitativa. Obtenido de http://www.ustatunja.edu.co/cong/images/ curso/guba_y_lincoln_2002.pdf

Faustino, J; Jiménez, F. (2005). El modelo de cogestión de cuencas hidrográficas en América Central. Disponible en: www.portalcuencas.net

Gómez, S. (2003). Análisis de vulnerabilidad con énfasis en sequía en la subcuenca del Río Aguas Calientes, Somoto, Nicaragua. Tesis Mag. Sc. Turrialba, CR, CATIE. $91 \mathrm{p}$.

INETER. (2012). DIRECCION GENERAL DE METEOROLOGIA. Obtenido de http://servmet.ineter.gob.ni/Meteorologia/climadenicaragua.php

Jiménez, F. (2005). Conceptos básicos en manejo de cuencas. Turrialba, Costa Rica: CATIE, 7 P.

Kammerbaver, H., León, J., Castellón, N., Gómez, S., \& Prins, C. (2011). Modelo de cogestión adaptativa de. Recursos Naturales y Ambiente, 6. MARENA (Ministerio del Ambiente y los Recursos Naturales). (2003). Informe del estado ambiental en Nicaragua. Managua.

Marin, A. M., Fernandez, D. N., Martínez, A. A., \& Suárez, D. d. (2006). Calidad del Agua en la parte alta de las Cuencas Jusn Cojo y el Salado. Revista Facultad Nacional de Agronomía, 16.

Padrana, J. Á. (2018). Criterios de calidad y gestión del agua potable. Madrid: UNED - Universidad Nacional de Educación a Distancia.

Parra, O. 2009. Gestión Integrada de Cuencas Hidrográficas. Centro de Ciencias Ambientales, Universidad de Concepción, Chile.

Solomon, E. P., R, M. L., \& W., B. y. (2013). Biología. Mexico: Cengage Learnig. Toledo, A., Barragán, E., \& Ortiz, J. (30 de Noviembre de 2005). Sustentabilidad Patrimonial de la Cuenca del Río Tepalcatepec. Obtenido de Turrialba: http://www.colmich.edu.mx/docencia/cer/colectivos/tepalcatepec/ protocolo.htm

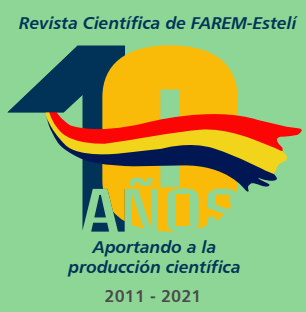

